\title{
Caspase-14, a keratinocyte specific caspase: mRNA splice variants and expression pattern in embryonic and adult mouse
}

Dear Editor,

The stratum corneum, composed of tough, rigid, interlocking keratinocytes, serves as an essential barrier between terrestial organisms and their environment. At the transition from the living layers of the epidermis to the stratum corneum, the cells of the epidermis lose their nuclei and other organelles, rearrange their cytoskeletal network, and cease metabolic activity. The regulatory and proteolytic events that coordinate the tightly controlled process of epidermal terminal differentiation are poorly understood. Caspases are conserved proteases with a vast repertoire of substrates including structural elements (nuclear lamin, actin, fodrin, keratin 18, plectin), repair elements (polyadenyl ribophosphorylase, DNA protein kinase $\mathrm{C}$ ), and regulatory elements (sterol regulatory element binding proteins, Rb, NF- $\kappa \mathrm{B}$ ), to list a few. ${ }^{1}$ Caspases have been shown to participate in lens epithelial differentiation $^{2}$ and erythropoiesis, ${ }^{3}$ two tissues in addition to the epidermis where the anucleate terminally differentiated cell serves an important function before being shed or phagocytized.

To examine a potential role for caspases in epidermal terminal differentiation, we first determined which caspases are expressed in the epidermis. RNase protection assays (RPAs) were performed on total RNA isolated from mouse skin and cultured mouse epidermal keratinocytes (MEKs). Mouse epidermis and MEKs express caspase-2, $-3,-6,-8$, and -14 , but not caspase-1, $-7,-11$, or -12 . Caspase-9 was detected in epidermis and cultured keratinocytes by RTPCR. To compare levels of caspase expression between basal and differentiating keratinocytes, we utilized RNA from MEKs grown in low and high calcium medium respectively. ${ }^{4}$ Caspase-14 displayed high mRNA levels in low calcium conditions and appeared to be downregulated after growth for $48 \mathrm{~h}$ in high calcium medium. The other caspase members showed no difference in mRNA levels between basal and differentiating keratinocytes. Northern analysis of cultured MEKs confirmed these findings, demonstrating abundant caspase-14 mRNA in low calcium conditions which increased in the first $16 \mathrm{~h}$ after switching to high calcium, and then decreased over the next $48 \mathrm{~h}$. Caspase-14 is a newer member of the caspase family, and was identified independently by three groups ${ }^{5-7}$ two of which suggested that caspase-14 may be skin specific. Because caspase-14 was the only caspase expressed in epidermis that showed differential expression, and because of reports suggesting that caspase-14 may be skin specific, we proceeded to further investigate this interesting caspase.

RNA from mouse epidermis and MEKs was subjected to RT-PCR using primers designed to amplify the entire coding region of mouse caspase-14. Two cDNAs were reproducibly obtained, a prominent product at $750 \mathrm{bp}$ and a weaker band at $500 \mathrm{bp}$ (Figure 1A), which were cloned and sequenced. The third PCR product of $800 \mathrm{bp}$ was not consistently observed, and was therefore not further studied. The $750 \mathrm{bp}$ cDNA, denoted C14L (caspase-14 long), encodes a protein identical to the published cDNA sequence (Genbank accession \#AF092997). The smaller cDNA, denoted C14S (caspase-14 short), encodes a protein of 159 amino acids that contains the QACRG active site, followed by a unique sequence of 21 amino acids followed by a stop codon. Sequencing of the mouse caspase-14 gene indicates that $\mathrm{C} 14 \mathrm{~S}$ arises from alternative splicing, effectively skipping exons 5 and 6 (Figure 1B). This splice event leads to a frame shift and results in a stop codon, predicting a protein that lacks all of the small subunit of normal caspase-14. The presence of C14S mRNA in mouse skin and MEKs was confirmed by RPA. A mRNA splice variant has recently been reported for human caspase-14 (casp-14b), ${ }^{8}$ however in contrast to C14S, it is slightly larger than the major human caspase-14 transcript, and is detected in post-confluent cultured human keratinocytes.

To further study the expression pattern of caspase-14, we generated a peptide antibody from a sequence within the large subunit. In mouse skin, caspase-14 expression was limited to the upper granular layer and to the infundibulum of the hair follicle. In MEKs, caspase-14 protein is expressed in differentiating keratinocytes. The cleaved form of caspase-14 is detected in abundance in mouse epidermis, but is not seen in MEKs, even when cells are subjected to apoptotic stimuli such as UV irradiation and growth factor withdrawal. Our data confirm a recent report describing the expression pattern in human skin and cultured keratinocytes. ${ }^{9}$ As reported here, no processing of caspase-14 to its large and small subunits was observed in cultured keratinocytes, which occurs only in skin and lifted cultures where more complete differentiation occurs. The paradigm for most caspases is that they must be cleaved into their respective large and small subunits with subsequent tetramer formation to form an active enzyme. However, some large prodomain initiator caspases, namely caspase-9, demonstrate enzymatic activity in the presence of appropriate cofactors. Procaspase-14 demonstrates measurable activity using DEVD-AFC as a substrate, ${ }^{6}$ but because it is a short prodomain caspase, we predict that it requires cleavage for full activity. The factor(s) that activate caspase-14 and its in vivo substrates have yet to be determined. 
A.

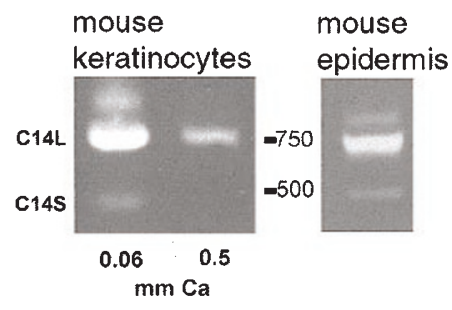

B.

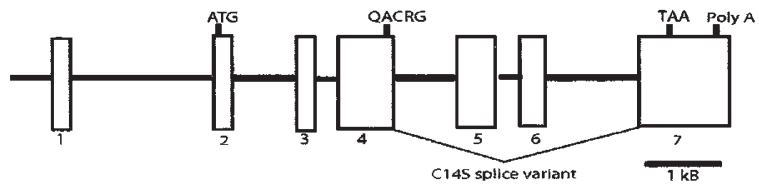

C.

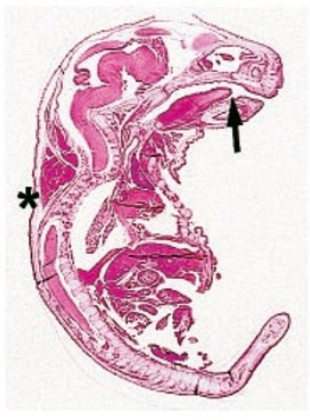

D.

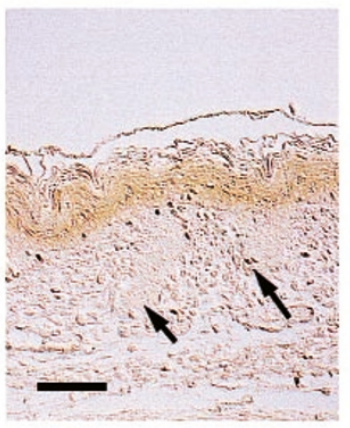

E.

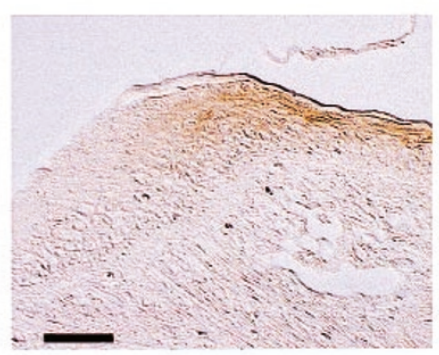

F.
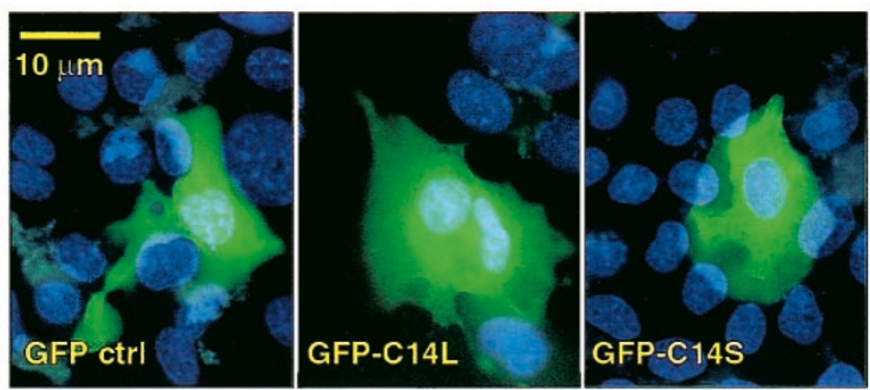

Figure 1 (A) Total RNA from mouse epidermis and mouse keratinocytes cultured in low and high calcium medium was subjected to RT-PCR using the following primers: 5'-GGACGAGGCGGACCGGCAGCT (sense) and 3'-TTATTGCAAATAGAGCTTCTTCTTCCG (antisense). Caspase-14 products obtained by RT-PCR were isolated by gel extraction, cloned into $p \mathrm{CR}^{\mathrm{R}} 3.1$ topo vector (Invitrogen, Carlsbad, CA, USA), and sequenced by dye terminator cycle sequencing (Perkin Elmer, Foster City, CA, USA). Mouse caspase-14 long (C14L) and caspase-14 short (C14S) clones were generated from both MEKs and mouse skin RNA: identical cDNAs were obtained from both sources. For expression in mouse keratinocytes, C14L and C14S obtained by RT-PCR were cloned into pcDNA3.1/NT-GFPTOPO ${ }^{\mathbb{R}}$ vector. (B) Genomic organization of the mouse caspase-14 reveals 7 exons. The C14S variant is derived by a splicing event that joins exons 4 and 7 , resulting in a frame shift and subsequent stop codon. The protein encoded by C14S is 159 amino acids and contains 21 amino acids after the conserved QACRG followed by a stop codon. (C, D, E) Whole mouse embryos at days 14,16 , and 18 were sectioned sagitally and stained for caspase-14 using an affinity purified polyclonal antibody to the following peptide sequence: DPGEELRGNEELGGDEE. No caspase-14 protein was detected at days 14 and 16 . At day 18, however, caspase-14 protein was seen in all cornifying epithelia. (C) is an H\&E stained section of a day 18 embryo which indicates the organs and tissues present at the time of caspase-14 immunostaining. (D) shows the expression of caspase-14 in dorsal skin ( ${ }^{*}$, asterisk C). (Bar=30 $\left.\mu \mathrm{M}\right)$. Caspase- 14 expression is found in the suprabasal layers of the epidermis and spares the follicular structures (arrows). (E) shows caspase-14 expression in the oral cavity (arrow, C). (Bar=20 $\mu \mathrm{M})$. In this view, the transition from the cornified epithelium of the lower lip to the mucosal epithelium of the oral cavity is shown. Caspase-14 is expressed in the cornifying epithelium of the lower lip, but is not expressed in the mucosal epithelium of the inner lip. (F) Overexpression of GFP-C14L and GFP-C14S does not lead to any morphologic changes in expressing cells. MEKs were transiently transfected with GFP and GFP fused to C14L and C14S at the amino terminus. The nuclei of transfected cells (green) show no evidence of chromatin condensation or shrinkage as evidenced by DAPI stain (blue). Immunoblot analysis of cells transfected with GFP-C14L with caspase-14 and GFP antibodies demonstrated no processing of transfected C14L (procaspase-14) in vitro (not shown)

During murine development, caspase-14 mRNA is detected briefly and in low abundance at embryonic day 7 , then is strongly expressed at day 15 to birth. ${ }^{6,7}$ To characterize the tissues where caspase-14 protein is expressed during embryogenesis, we immunostained sagital sections of whole embryos at day 14,16 , and 18 . Caspase-14 protein appeared between embryonic day 16 and 18 in cornified epithelium in the suprabasal layers (Figure 1C-E). Caspase-14 was not detected in any other organs, including other epithelia and mesenchymal tissues, 
nor was any caspase-14 detected in mucosal epithelia. Interestingly, in contrast to newborn and adult skin, caspase-14 expression was not restricted to the upper granular layer (Figure 1D), but was found throughout the suprabasal layers, indicating it may function during embryogenesis during epithelial maturation. Caspase-14 expression appears at the same time that murine skin begins to fully differentiate into a cornified epithelium, and is not expressed in noncornified epithelia (Figure 1E).

The high levels of caspase-14 mRNA found in MEKs with a basal phenotype appears to contradict the expression pattern of caspase-14 protein which immunolocalizes to the terminally differentiating layers of the skin. However, mRNA for caspase-14 increases within the first $16 \mathrm{~h}$ after inducing differentiation in MEKs before falling over the next $48 \mathrm{~h}$. One explanation for this observation is that once differentiation is induced, caspase-14 mRNA is no longer needed. Further, the cultures become nonviable after several days in high calcium, so another explanation is that due to terminal differentiation, many cellular functions are ending. In contrast to human keratinocytes, markers of differentiation in MEKs are not a function of confluence (personal communication, C Hull and P Fleckman), and therefore had no bearing on caspase-14 expression.

Splice variants for other caspases and proteins that regulate caspase function have been reported, ${ }^{10,11}$ and the products of such alternative messages tend to have antagonistic effects on the normal enzyme. To study the role of the $\mathrm{C} 14 \mathrm{~S}$, we generated GFP fusion proteins with C14L (full-length procaspase-14) and C14S (the 159 amino acid splice variant) and overexpressed them in MEKs. No morphologic evidence of apoptosis was seen in cells expressing C14L or C14S as determined by DAPI staining (Figure 1F). An equal percentage of cells demonstrated green fluorescence whether transfected with GFP alone or GFP-fused caspase-14, indicating that the lack of adverse effects on expressing cells was not due to cell loss. Some caspases will autoactivate when overexpressed in mammalian cells, however no processing of recombinant GFPC14L was observed in transfected MEKs grown in low or high calcium as determined by immunoblot analysis (not shown). The lack of processing of caspase-14, even when overexpressed in its native cell type, lends further evidence to its requirement for conditions of terminal differentiation for cleavage to occur. Whether processing is an absolute requirement for activity has yet to be determined. Further, the lack of apoptotic morphology in C14L and C14S overexpressing cells concurs with the lack of caspase-14 activation seen in conditions that induce apoptosis.

In summary, we have identified a unique splice variant of mouse caspase-14 that is differentially expressed in MEKs. The function of C14S is currently unknown. In the case of caspase-2, the short splice variant is postulated to have an antagonistic effect on full-length caspase-2, possibly by forming non-functional tetramers. The short splice variant of caspase-14, based on its protein sequence, may function in a similar manner, however further studies are needed to ascertain the function of both transcripts in vivo. During embryogenesis, caspase-14 expression correlates with the onset of epidermal stratification, and is found only in cornified epithelia. Studies are ongoing in our laboratory to determine the factor(s) that activate caspase-14, the in vivo substrate(s) of caspase-14, and the function of the short splice variant of caspase-14. We predict that caspase-14 functions during epidermal terminal differentiation to cleave structural proteins or activate enzymes found in the granular layer that participate in the formation of the stratum corneum.

\section{Acknowledgements}

The authors would like to gratefully acknowledge $\mathrm{Dr}$ Andrew Farr, Department of Biologic Structure, University of Washington, Seattle, WA, for supplying embryonic mice. This work was supported by the NIH (PO1 AM21557), the Dermatology Foundation and the Odland Endowment Fund of the Division of Dermatology, Department of Medicine, University of Washington.

MK Kuechle ${ }^{*, 1,2}$, HM Predd ${ }^{2}$, Pleckman $^{2}$, BA Dale Pl, $^{1,3,4}$ and RB Presland ${ }^{1,2}$

1 Department of Oral Biology, University of Washington, Seattle, Washington, WA 98195, USA

2 Department of Medicine (Dermatology), University of Washington, Seattle, Washington, WA 98195, USA

${ }^{3}$ Department of Periodontics, University of Washington, Seattle, Washington, WA 98195, USA

4 Department of Biochemistry, University of Washington, Seattle, Washington, WA 98195, USA

* Corresponding author: Dr Melanie K Kuechle, Box 357132, University of Washington, Seattle WA, 98195-7132, USA. Tel: (206)-543-1595; Fax: (206)-685-3162; E-mail: kuechles@u.washington.edu

1. Porter A, Ng P and Janicke R (1997) Bioessays 19: 501-507

2. Ishizaki Y, Jacobson M and Raff M (1998) J. Cell. Biol. 140: 153-158

3. DeMaria $R$ et al. (1999) Nature 401: 489-493

4. Hager B, Bickenbach J and Fleckman P (1999) J. Invest. Dermatol. 112: 971 976

5. Ahmad M et al. (1998) Cancer Res. 58: 5201-5205

6. Hu S et al. (1998) J. Biol. Chem. 273: 29648-29653

7. VandeCraen M et al. (1998) Cell Death Differ. 5: 838-846

8. Eckhart L et al. (2000) Biochem. Biophys. Res. Comm. 277: 655-659

9. Lippens S et al. (2000) Cell Death Differ. 7: 1218-1224

10. Wang L et al. (1994) Cell 78: $739-750$

11. Seol D-W and Billiar T (1999) J. Biol. Chem., 274: 2072-2076 